\title{
QUASIPERIODIC AND MIXED COMMUTATOR FACTORIZATIONS IN FREE PRODUCTS OF GROUPS
}

\author{
SERGEI V. IVANOV AND ANTON A. KLYACHKO
}

\begin{abstract}
It is well known that a nontrivial commutator in a free group is never a proper power. We prove a theorem that generalizes this fact and has several worthwhile corollaries. For example, an equation $\left[x_{1}, y_{1}\right] \ldots\left[x_{k}, y_{k}\right]=$ $z^{n}$, where $n \geq 2 k$, in the free product $\mathcal{F}$ of groups without nontrivial elements of order $\leq n$ implies that $z$ is conjugate to an element of a free factor of $\mathcal{F}$. If a nontrivial commutator in a free group factors into a product of elements which are conjugate to each other then all these elements are distinct.
\end{abstract}

\section{INTRODUCTION}

It was observed by Schützenberger 31 that a nontrivial commutator in a free group is never a proper power. This result was generalized in different directions: for values of other than commutator words on free groups by Baumslag and Steinberg [1], for values of commutators on free products of groups by Comerford, Edmunds, and Rosenberger [7, and for values of commutators on small cancellation groups by Frenkel and the second author [12]. Our Theorem could be considered as one more such a generalization.

Theorem. Let $G_{\alpha}, \alpha \in I$, be torsion free groups and let $\mathcal{F}=\underset{\alpha \in I}{*} G_{\alpha}$ denote their free product. Suppose that

$$
c_{1} \ldots c_{k} d_{1} \ldots d_{\ell}=h_{1}^{n_{1}} \ldots h_{m}^{n_{m}}
$$

in $\mathcal{F}$, where $c_{1}, \ldots, c_{k}, d_{1}, \ldots, d_{\ell}, h_{1}, \ldots, h_{m}$ are elements of $\mathcal{F}$ such that $c_{i}$ are commutators, $d_{i^{\prime}}$ are conjugate to elements of free factors of $\mathcal{F}, h_{j}$ are conjugate to each other and are not conjugate to elements of free factors of $\mathcal{F}$, and $n_{j}$ are positive integers. Then

$$
\sum_{j=1}^{m}\left(n_{j}-1\right) \leq 2 k+\ell-2 .
$$

In addition, the same statement holds for any free product $\mathcal{F}$ of groups with torsion whenever the order of every letter of a cyclically reduced word conjugate to $h_{1}$ is greater than $\sum_{j=1}^{m} n_{j}$.

M. Culler 8 discovered that, in the free group $F(a, b)$ with free generators $a, b$, the cube $[a, b]^{3}$ of the commutator $[a, b]:=a^{-1} b^{-1} a b$ is a product of two commutators,

$$
[a, b]^{3}=\left[a^{-1} b a, a^{-2} b a b^{-1}\right]\left[b a b^{-1}, b^{2}\right] .
$$

Moreover, $[a, b]^{n}$ is a product of $k$ commutators whenever $n \leq 2 k-1$, see $[8$.

2010 Mathematics Subject Classification. Primary 20E06, 20F06, 20F70, 57M07.

The work of the second author was supported by the Russian Foundation for Basic Research, project 15-01-05823. 
Comerford, Comerford, and Edmunds [6] proved that a nontrivial product of two commutators in a free group cannot be more than a third power, i.e., the equality $\left[x_{1}, y_{1}\right]\left[x_{2}, y_{2}\right]=z^{n}$, where $n \geq 4$, in a free group implies that $z=1$.

The authors of [6] conjectured that, in a free group, the Culler's bound $n \leq 2 k-1$ is sharp. In other words, the Comerford-Comerford-Edmunds conjecture asserts that, in a free group, the equality $\left[x_{1}, y_{1}\right] \ldots\left[x_{k}, y_{k}\right]=z^{n}$, where $n \geq 2 k$, implies that $z=1$. This conjecture was proven by Duncan and Howie [9, Theorem 3.3] by establishing that, in the free product $A * B$ of two locally indicable groups $A, B$, the equality $\left[x_{1}, y_{1}\right] \ldots\left[x_{k}, y_{k}\right]=z^{n}$, where $n \geq 2 k$, implies that $z$ is conjugate to an element of $A$ or $B$. Our Theorem implies, in particular, that a similar result holds true for the free product of torsion free groups and for the free product of groups with torsion bounded below. Note that, in the case of small torsion, a similar result is no longer true: in the infinite dihedral group $\langle c\rangle_{2} *\langle d\rangle_{2}$, every power of the commutator $[c, d]$ is also a commutator.

Corollary 1. Let $\mathcal{F}=\underset{\alpha \in I}{*} G_{\alpha}$ be the free product of groups $G_{\alpha}, \alpha \in I$, that contain no nontrivial elements of order $\leq n$. Then an equality $\left[x_{1}, y_{1}\right] \ldots\left[x_{k}, y_{k}\right]=z^{n}$, where $n \geq 2 k$, in $\mathcal{F}$ implies that $z$ is conjugate to an element of a free factor of $\mathcal{F}$.

We remark that Corollary 1 is similar to a recent result of Chen [2, Corollary 3.7], however, neither Corollary 1 follows from [2, Corollary 3.7] nor [2, Corollary 3.7] follows from Corollary 1

We now state more corollaries of our Theorem.

Corollary 2. Let $\mathcal{F}$ be the free product of torsion free groups $G_{\alpha}, \alpha \in I$. Suppose that

$$
c_{1} \ldots c_{k} d_{1} \ldots d_{\ell}=h_{1} \ldots h_{m}
$$

in $\mathcal{F}$, where $c_{1}, \ldots, c_{k}, d_{1}, \ldots, d_{\ell}, h_{1}, \ldots, h_{m}$ are elements of $\mathcal{F}$ such that $c_{i}$ are commutators, $d_{i}$ are conjugate to elements of free factors of $\mathcal{F}$, and $h_{i}$ are conjugate to each other and are not conjugate to elements of free factors of $\mathcal{F}$. Then no element occurs in the sequence $h_{1}, \ldots, h_{m}$ more than $2 k+\ell-1$ times.

Corollary 3. Suppose $F$ is a free group and $c=h_{1} \ldots h_{m} \neq 1$ in $F$, where $c, h_{1}, \ldots, h_{m} \in F$ are such that $c$ is a commutator and $h_{1}, \ldots, h_{m}$ are conjugate to each other. Then $h_{1}, \ldots, h_{m}$ are all distinct.

More generally, suppose that $c_{1} \ldots c_{k}=h_{1} \ldots h_{m} \neq 1$ in a free group $F$, where $c_{1}, \ldots, c_{k}, h_{1}, \ldots, h_{m} \in F$ are such that $c_{1}, \ldots, c_{k}$ are commutators and $h_{1}, \ldots, h_{m}$ are conjugate to each other. Then no element occurs in the sequence $h_{1}, \ldots, h_{m}$ more than $2 k-1$ times.

Corollary 4. Let $A * B$ be the free product of torsion free groups $A, B$. Then no nontrivial element $a \in A$ is a product of elements that are conjugate to each other and are not conjugate to an element of $A$.

More generally, suppose that $a_{1} b_{1} \ldots a_{\ell} b_{\ell}=h_{1} \ldots h_{m} \neq 1$ in $A * B$, where $a_{1}, \ldots, a_{\ell} \in A \backslash\{1\}, b_{1}, \ldots, b_{\ell} \in B \backslash\{1\}$, and $h_{1}, \ldots, h_{m} \in A * B$ are conjugate to each other and are not conjugate to an element of $A \cup B$. Then no element occurs in the sequence $h_{1}, \ldots, h_{m}$ more than $2 \ell-1$ times.

Let $w$ be an element of the free product $\mathcal{F}$ of groups $G_{\alpha}, \alpha \in I$. A mixed commutator factorization for $w$ is an equality in $\mathcal{F}$ of the form

$$
w=c_{1} \ldots c_{k} d_{1} \ldots d_{\ell}
$$


where $c_{i}$ are commutators and $d_{j}$ are conjugate to elements of free factors of $\mathcal{F}$. The mixed genus $\operatorname{mg}(w)$ of $w$ is defined to be a minimal integer $s$ such that $s=2 k+\ell$ over all mixed commutator factorizations (11) for $w$.

For example, if $\operatorname{mg}(w) \leq 1$ then $w$ is conjugate to an element of a free factor of $\mathcal{F}$ and if $\operatorname{mg}(w)=2$ then $w$ is a commutator or a product of two elements conjugate to nontrivial elements of free factors of $\mathcal{F}$.

We remark that Culler $[8$ introduced the genus $g(w)$ for an element $w$ of the free product $A * B$ of two groups $A, B$ as a minimal number of commutators needed to write $w$ as the product of these commutators or $g(w):=\infty$ if $w$ is not a product of commutators. Culler 8 gave an algorithm that computes the genus $g(w)$ of $w$ whenever the genera of elements can be computed in free factors $A, B$. The genus $g(w)$ can be defined in the same fashion for an element $w$ of an arbitrary free product $\mathcal{F}$ of groups.

Let a free group $F$ be considered as the free product of its cyclic subgroups. Grigorchuk and Kurchanov [14] defined the width $h(w)$ of an element $w$ of $F$ as a minimal number of elements that are conjugate to elements of free factors of $F$ and that are needed to write $w$ as their product. Grigorchuk and Kurchanov [14] gave an algorithm that computes the width $h(w)$ of $w \in F$, see also [17, [30. The width $h(w)$ can be defined in the same manner for an element $w$ of an arbitrary free product $\mathcal{F}$ of groups.

It is worthwhile to note that our definition of the mixed genus $\operatorname{mg}(w)$ of an element $w$ of an arbitrary free product $\mathcal{F}$ combines the foregoing two definitions and the number $\operatorname{mg}(w)$ satisfies the inequalities $\operatorname{mg}(w) \leq 2 g(w)$ and $\operatorname{mg}(w) \leq h(w)$. However, it is not clear how to algorithmically compute the mixed genus $\operatorname{mg}(w)$ even for elements of a free group.

A quasiperiodic factorization for an element $w$ of the free product $\mathcal{F}$ of groups $G_{\alpha}, \alpha \in I$, is an equality in $\mathcal{F}$ of the form

$$
w=h_{1}^{n_{1}} \ldots h_{m}^{n_{m}}
$$

where $h_{1}, \ldots, h_{m}$ are conjugate to each other and are not conjugate to an element of a free factor $G_{\alpha}, n_{1}, \ldots, n_{m}$ are positive integers, and $m \geq 1$.

The quasiperiodicity $\mathrm{qp}(w)$ of $w$ is defined to be a maximal integer $r$ such that $r=\sum_{j=1}^{m}\left(n_{j}-1\right)$ over all quasiperiodic factorizations (2) for $w$ if there are such factorizations and the set of such $r$ is bounded above. If the set of such $r$ is not bounded above, we set $\mathrm{qp}(w):=+\infty$ and if there are no such factorizations for $w$, we set $\mathrm{qp}(w):=-\infty$.

It is clear that, for every $w \in \mathcal{F}$ such that $w$ is not conjugate to an element of a free factor, we have $\mathrm{qp}(w) \geq 0$. As another example, consider two elements $u, v \in \mathcal{F}$ that are conjugate and are not conjugate to an element of a free factor of $\mathcal{F}$. Then $\operatorname{qp}\left(u^{4} v^{2}\right) \geq 4$ and $\operatorname{qp}\left(u^{3} v u v\right) \geq 3$ as $u^{3} v u v=u^{4} v^{u} v$, where $v^{u}:=u^{-1} v u$.

Note that if $w=h_{1} \ldots h_{m}$ in $\mathcal{F}$, where $h_{1}, \ldots, h_{m}$ are conjugate to each other and are not conjugate to an element of a free factor $G_{\alpha}$, and $s$ elements among $h_{1}, \ldots, h_{m}$ are equal each other, then $\mathrm{qp}(w) \geq s-1$. Indeed, we can apply the identity $u v=v u^{v}$ and rearrange the factors $h_{1}, \ldots, h_{m}$ in such a way that the equal $s$ factors would form an sth power. This observation, in particular, implies that, if the free product $A * B$ has torsion, then $\mathrm{qp}(1)=+\infty$. Indeed, if an element $a \in A$ has order $m>1$ and $b \in B$ is nontrivial then

$$
1=[a, b][a, b]^{a^{-1}} \ldots[a, b]^{a^{1-m}}=\left([a, b][a, b]^{a^{-1}} \ldots[a, b]^{a^{1-m}}\right)^{2016} .
$$


These equalities mean that $\mathrm{qp}(1)=\mathrm{qp}([a, b])=+\infty$. (It is not clear what could be $\mathrm{qp}(a), \mathrm{qp}(a b)$ in this situation.) On the other hand, for free products of groups without torsion we have a nicer situation.

Corollary 5. Let $\mathcal{F}$ be the free product of torsion free groups $G_{\alpha}, \alpha \in I$. Then, for every $w \in \mathcal{F}$, the quasiperiodicity $\mathrm{qp}(w)$ of $w$ satisfies $\mathrm{qp}(w) \leq \operatorname{mg}(w)-2<+\infty$. Furthermore, $\operatorname{qp}(w)=-\infty$ if and only if $w$ is conjugate to an element of a free factor of $\mathcal{F}$, otherwise, $\mathrm{qp}(w) \geq 0$ is finite.

We remark that the bound $\mathrm{qp}(w) \leq \operatorname{mg}(w)-2$ of Corollary 5 is sharp as follows from the equality

$$
(a b)^{n}=a^{n} b^{a^{n-1}} b^{a^{n-2}} \ldots b^{a} b
$$

that proves that if $a \in A, b \in B$ are nontrivial then $\mathrm{qp}\left((a b)^{n}\right) \geq n-1$ and $\operatorname{mg}\left((a b)^{n}\right) \leq n+1$. The sharpness of the bound $\mathrm{qp}(w) \leq \operatorname{mg}(w)-2$ also follows from the Culler's observation [8] that $[a, b]^{n}$ is a product of $k$ commutators whenever $n \leq 2 k-1$.

Our arguments utilize diagrams over free products of groups and are based on a car-crash lemma of 19, 20, 21, see also [10, that has had quite a few applications in group theory, see [3], 44, [5], [11, [12, [13], [18, [22], 23], 24], 25], 26], 27].

In Sect. 2, we define diagrams over free products of groups and prove a lemma on geometric meaning of the mixed genus. In Sect. 3, we state a car-crash lemma. Sect. 4 contains the proof of our Theorem.

\section{Preliminaries}

Suppose that $S$ is an oriented compact closed surface. Note that $S$ need not be connected.

A map on $S$ is a finite 2-complex $\Delta$ embedded into $S$. We call $S$ the underlying surface for $\Delta$, denoted $S=S(\Delta)$. If the embedding of $\Delta$ into $S$ is surjective, i.e., $\Delta$ has no boundary, we say that the map $\Delta$ is closed.

The set of $i$-cells of a finite 2 -complex $\Delta$ is denoted $\Delta(i), i=0,1,2$. The closures of $i$-cells of $\Delta$ for $i=0,1,2$ are called vertices, edges, faces, resp. The 1-skeleton of $\Delta$, consisting of vertices and edges, is a graph denoted $\Delta[1]$.

If $F$ is a face of a map $\Delta$ then a boundary path $\partial F$ of $F$ is oriented in positive, i.e., in counterclockwise, direction. Recall that $S(\Delta)$ is oriented. If $\partial F=e_{1} e_{2} \ldots e_{k}$, where $e_{1}, e_{2}, \ldots, e_{k}$ are oriented edges, then the subpaths $e_{1} e_{2}, e_{2} e_{3}, \ldots, e_{k} e_{1}$ of $\partial F$ are called corners of $F$. If $e_{i} e_{i+1}$ is a corner of $F$ then the terminal vertex of $e_{i}$ is called the vertex of $e_{i} e_{i+1}$ and is denoted $\nu\left(e_{i} e_{i+1}\right)$.

If $e$ is an oriented edge of a 2-complex $\Delta$ then $e_{-}, e_{+}$denote the initial, terminal, resp., vertices of $e$. By $e^{-1}$ we mean the edge with opposite to $e$ orientation. If $p=e_{1} \ldots e_{k}$ is a path in $\Delta$, where $e_{1}, \ldots, e_{k}$ are oriented edges, then the initial and terminal vertices of $p$ are defined by $p_{-}:=\left(e_{1}\right)_{-}$and $p_{+}:=\left(e_{k}\right)_{+}$, resp., and $p^{-1}:=e_{k}^{-1} \ldots e_{1}^{-1}$.

Let $C(\Delta)$ denote the set of all corners of faces of a map $\Delta$ and let $A * B$ be the free product of two nontrivial groups $A, B$, where $A \cap B=\{1\}$. A map $\Delta$ is called a diagram over $A * B$ if $\Delta$ is equipped with two labeling functions

$$
\varphi: C(\Delta) \rightarrow A \cup B, \quad \theta: \Delta(0) \rightarrow\{A, B\}
$$

and the following conditions are satisfied. 
(D1) If $u, v$ are two vertices of $\Delta$ connected by an edge then $\theta(u) \neq \theta(v)$. In particular, $\Delta[1]$ is a biparite graph.

(D2) For every corner $e e^{\prime} \in C(\Delta)$ such that $\theta\left(\nu\left(e e^{\prime}\right)\right)=A$, we have $\varphi\left(e e^{\prime}\right) \in A$ and, for every corner $e e^{\prime} \in C(\Delta)$ such that $\theta\left(\nu\left(e e^{\prime}\right)\right)=B$, we have $\varphi\left(e e^{\prime}\right) \in$ $B$.

We remark that our definition of a diagram over $A * B$ is different from the definitions of diagrams over free products of groups used in books [28, 29] and is similar to the definition introduced in Howie's articles [15, [16].

Let $F$ be a face of a diagram $\Delta$ over $A * B$ and let $\partial F=e_{1} e_{2} \ldots e_{k}$, where $e_{1}, e_{2}, \ldots, e_{k}$ are oriented edges, be a boundary path of $F$. A label $\varphi(\partial F)$ of $F$ is defined by setting

$$
\varphi(\partial F):=\varphi\left(e_{1} e_{2}\right) \varphi\left(e_{2} e_{3}\right) \ldots \varphi\left(e_{k} e_{1}\right),
$$

i.e., $\varphi(\partial F)$ is the product of consecutive, in positive direction, $\varphi$-labels of corners of $F$. It is clear that $\varphi(\partial F)$ is a word over the alphabet $A \cup B$ and $\varphi(\partial F)$ is defined up to a cyclic permutation.

Let $p=e_{i} e_{i+1} \ldots e_{i+\ell}$ be a subpath of a boundary path $\partial F$ of a face $F$, where indices are modulo $k=|\partial F|$. We define the label $\varphi(p)$ of $p$ to be the word

$$
\varphi(p):=\varphi\left(e_{i} e_{i+1}\right) \varphi\left(e_{i+1} e_{i+2}\right) \ldots \varphi\left(e_{i+\ell-1} e_{i+\ell}\right) .
$$

If $v \in \Delta(0)$ is a vertex in the interior of a diagram $\Delta$ over $A * B$, i.e., $v \notin \partial \Delta$, then a label $\varphi(v)$ of $v$ is the product of $\varphi$-labels of consecutive, in negative direction, corners whose vertex is $v$. We say that $v$ is an $A$-vertex if $\theta(v)=A$ and $v$ is a $B$ vertex if $\theta(v)=B$. It is clear from the definitions that $\varphi(v) \in A$ if $v$ is an $A$-vertex and $\varphi(v) \in B$ if $v$ is a $B$-vertex. It is also clear that $\varphi(v)$ is defined up to conjugation in $A$ or $B$. If $\varphi(v)$ is defined and $\varphi(v)=1$ in $A$ or in $B$, depending on type of $v$, then we say that $v$ is a regular vertex. If $\varphi(v)$ is defined and $\varphi(v) \neq 1$ in $A$ or $B$ then $v$ is called an irregular vertex. Note that a label $\varphi(v)$ is not defined for a vertex $v$ on the boundary $\partial \Delta$ of $\Delta$.

We remark that similar diagrams were considered in [15], [16], 19], 27] and some other papers but our definitions are slightly different.

For example, the diagram depicted in Fig. 1 has a torus as the underlying surface and it is drawn as a rectangle with opposite sides to be identified. This diagram contains two vertices, three edges, one face, and three corners with $\varphi$-label $a \in A$ and three corners with $\varphi$-label $b \in B$. If the vertices are regular, then $a^{3}=1$ in $A$ and $b^{3}=1$ in $B$. The label of the face is $(a b)^{3}$. This diagram demonstrates that if $a \in A$ and $b \in B$ have order 3 then $(a b)^{3}$ is a commutator. A complete description of commutators in a free product of groups that are not conjugate to elements of free factors and are proper powers is given in 7 .

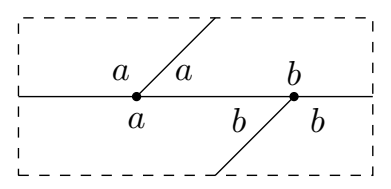

Fig. 1

We call a diagram $\Delta$ over $A * B$ reduced if $\Delta$ has no face with a corner whose $\varphi$-label is 1 . 
The extended genus $\operatorname{eg}(\Delta)$ of a diagram $\Delta$ over $A * B$ is defined by

$$
\operatorname{eg}(\Delta):=2-\chi(\Delta)+r_{0}(\Delta)
$$

where $\chi(\Delta)=|\Delta(0)|-|\Delta(1)|+|\Delta(2)|$ is the Euler characteristic of $\Delta$ and $r_{0}(\Delta)$ is the number of irregular vertices in $\Delta$.

We consider elements of the free product $A * B$ as words over the alphabet $A \cup B$, where $A \cap B=\{1\}$, whose elements are called letters. A word $w=a_{1} \ldots a_{\ell}$, where $a_{1}, \ldots, a_{\ell} \in A \cup B$ are letters, is called reduced if $w$ is nonempty, none of the letters $a_{1}, \ldots, a_{\ell}$ is 1 and, for every $i$, the letters $a_{i}, a_{i+1}$ do not belong to the same free factor of $A * B$. The length of a word $w$ is the number of letters in $w$, denoted $|w|$. A word $w$ is cyclically reduced if $w$ is nonempty and $w^{2}$ is reduced.

The definitions of reduced and cyclically reduced words that represent elements of an arbitrary free product $\mathcal{F}=\underset{\alpha \in I}{*} G_{\alpha}$ of nontrivial groups $G_{\alpha}$ are analogous.

If $u, w$ are two words over $A \cup B$, then $u \equiv w$ means the literal or letter-by-letter equality of words. If words $u, w$ are equal as elements of $A * B$, we write $u \stackrel{*}{=} w$.

If $w$ is a word over $A \cup B$, we let $\delta_{1}(w)$ denote the word obtained from $w$ by deletion of all occurrences of the letter $1 \in A \cup B$. By writing $w \equiv_{1} u$ we mean that $\delta_{1}(w) \equiv \delta_{1}(u)$. If $a, b \in A$ then by writing $a \stackrel{A}{=} b$ we mean that $a=b$ in $A$. Similarly, the notation $a \stackrel{B}{=} b$ means that $a, b \in B$ and $a=b$ in $B$.

We mention without proof that the mixed genus $\operatorname{mg}(w)$ of a cyclically reduced word $w$ over $A \cup B$ is equal to the minimal extended genus $\operatorname{eg}(\Delta)$ of a reduced closed diagram $\Delta$ over $A * B$ that contains a single face whose label is the word $w$. In this paper we need only the inequality $\operatorname{mg}(w) \geq \operatorname{eg}(\Delta)$ that follows from Lemma 1 below. We also remark that the inequality $\operatorname{mg}(w) \geq \operatorname{eg}(\Delta)$ of Lemma 1 is actually an equality but we will not need this equality either.

Lemma 1. Suppose that $u_{1}, \ldots, u_{m}$ are nonempty cyclically reduced words over the alphabet $A \cup B$. Then the minimal mixed genus $\operatorname{mg}(w)$ of an element $w \in A * B$ of the form $w \stackrel{*}{=} s_{1} u_{1} s_{1}^{-1} \ldots s_{m} u_{m} s_{m}^{-1}$ satisfies $\operatorname{mg}(w) \geq \operatorname{eg}(\Delta)$, where $\operatorname{eg}(\Delta)$ is the minimal extended genus $\operatorname{eg}(\Delta)$ of a reduced closed diagram $\Delta$ over $A * B$ that contains precisely $m$ faces whose labels are the words $u_{1}, \ldots, u_{m}$.

Proof. Suppose that $w_{0}$ is a word of a minimal mixed genus $n=\operatorname{mg}\left(w_{0}\right)$ among all words $w$ of the form

$$
w \equiv s_{1} u_{1} s_{1}^{-1} \ldots s_{m} u_{m} s_{m}^{-1},
$$

where each $s_{i} \in A * B$ is a reduced word or $s_{i} \equiv 1$. Since $n=\operatorname{mg}\left(w_{0}\right)$, there is a factorization for $w_{0}$ of the form

$$
w_{0} \stackrel{*}{=}\left[v_{1}, t_{1}\right] \ldots\left[v_{k}, t_{k}\right] d_{1} \ldots d_{\ell},
$$

where $n=2 k+\ell$, for every $i, v_{i}, t_{i}$ are reduced words, and, for each $j, d_{j} \equiv$ $d_{j, 1} d_{j, 0} d_{j, 1}^{-1}, d_{j, 0} \in A \cup B, d_{j, 0} \neq 1, d_{j, 1}$ is a reduced word or $d_{j, 1} \equiv 1$.

Consider the word

$$
s_{m} u_{m}^{-1} s_{m}^{-1} \ldots s_{1} u_{1}^{-1} s_{1}^{-1} v_{1}^{-1} t_{1}^{-1} v_{1} t_{1} \ldots v_{k}^{-1} t_{k}^{-1} v_{k} t_{k} d_{1} \ldots d_{\ell} .
$$

Let $\Delta_{0}$ be a diagram over $A * B$ that consists of a single face $H$ whose boundary path $\partial H$ has the following factorization

$$
\partial H=p_{1} q_{1} p_{2} q_{2} \ldots p_{L} q_{L},
$$


where $L=3 m+4 k+\ell, p_{1}, q_{1}, \ldots p_{L} q_{L}$ are subpaths of $\partial H$, and the sequence of words $\varphi\left(p_{1}\right), \ldots, \varphi\left(p_{L}\right)$ is identical to the sequence of subwords $s_{1}, u_{1}^{-1}, s_{1}^{-1}, \ldots, d_{\ell}$ distinguished in the word (4). The paths $q_{1}, \ldots, q_{L}$ have labels equal to powers of the letter $1 \in A \cup B$ and $\left|q_{i}\right|=2$ or $\left|q_{i}\right|=3$, hence, $\varphi\left(q_{i}\right) \equiv 1^{\left|q_{i}\right|-1}$ with $\left|q_{i}\right|-1 \geq 1$. The $\varphi$-label of a corner of $H$ whose vertex is $\left(p_{i}\right)_{-}$or $\left(p_{i}\right)_{+}$is also 1 . It is easy to see that we can assign $\theta$-labels to corners of $H$ so that both properties (D1)-(D2) hold true and $\Delta_{0}$ is indeed a diagram over $A * B$. Note that the choice between $\left|q_{i}\right|=2$ or $\left|q_{i}\right|=3$ depends on $\theta\left(\left(p_{i}\right)_{+}\right)$and $\theta\left(\left(p_{i+1}\right)_{-}\right)$, here indices are modulo $L$.

Let $x$ be one of the subwords $s_{m}, u_{m}^{-1}, s_{m}^{-1}, \ldots, d_{\ell}$ distinguished in the word (41) and let $p(x)$ denote the corresponding path among $p_{1}, p_{2}, \ldots, p_{L}$ such that $\varphi(p(x)) \equiv x$, i.e., we assume that $p\left(s_{m}\right)=p_{1}, p\left(u_{m}^{-1}\right)=p_{2}, \ldots, p\left(d_{\ell}\right)=p_{L}$.

We now make some surgeries over $\Delta_{0}$. We remark that $\theta$-labels of vertices never change under these surgeries.

Observe that the subpath $p\left(d_{j}\right)$ of $\partial H$ has even length because $d_{j} \equiv d_{j, 1} d_{j, 0} d_{j, 1}^{-1}$, where $d_{j, 0} \in A \cup B$ and $d_{j, 0} \neq 1, d_{j, 1}$ is a reduced word or $d_{j, 1} \equiv 1$. Hence, there is a factorization $p\left(d_{j}\right)=p_{1}\left(d_{j}\right) p_{2}\left(d_{j}\right)$, where $\left|p_{1}\left(d_{j}\right)\right|=\left|p_{2}\left(d_{j}\right)\right|$ and $\varphi\left(p_{1}\left(d_{j}\right)\right) \equiv$ $\varphi\left(p_{2}\left(d_{j}\right)\right)^{-1}$. Therefore, by identifying $p_{1}\left(d_{j}\right)$ and $p_{2}\left(d_{j}\right)^{-1}$ for each $j=1, \ldots, \ell$, within $H$, so that the subpath $p\left(d_{j}\right)$ of $\partial H$ turns into $\bar{p}_{1}\left(d_{j}\right) \bar{p}_{1}\left(d_{j}\right)^{-1}$, see Fig. 2 , we obtain a diagram $\Delta_{1}$ over $A * B$ with a single face, still denoted $H$, and $\ell$ irregular vertices $\left(\bar{p}_{1}\left(d_{j}\right)\right)_{+}, j=1, \ldots, \ell$. Note that all vertices of the paths $\bar{p}_{1}\left(d_{j}\right)$, except for their end vertices $\left(\bar{p}_{1}\left(d_{j}\right)\right)_{-} \in \partial \Delta_{1}$ and $\left(\bar{p}_{1}\left(d_{j}\right)\right)_{+}$are regular.

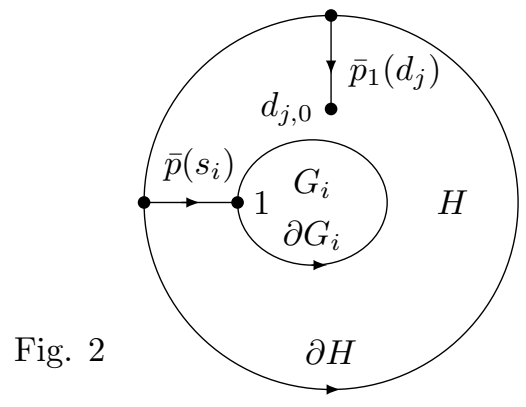

Our next step is to identify, for every $i=1, \ldots, m$, the path $p\left(s_{i}\right)$ with $p\left(s_{i}^{-1}\right)^{-1}$ within $H$, getting thereby a new path $\bar{p}\left(s_{i}\right)$ and a new map $\Delta_{2}$ with $m+1$ faces $H, G_{1}, \ldots, G_{m}$ such that the boundary path $\left.\partial\right|_{\left(\bar{p}\left(s_{i}\right)\right)_{+}} G_{i}$ of $G_{i}$ starting at the vertex $\left(\bar{p}\left(s_{i}\right)\right)_{+}$is a subpath of $(\partial H)^{-1}$, see Fig. 2. We assign 1 as the $\varphi$-label to the corner of $G_{i}$ whose vertex is $\left(\bar{p}\left(s_{i}\right)\right)_{+}$and, to every other corner of $G_{i}$, we assign $\varphi$-label equal to the inverse of the $\varphi$-label of the corner of $H$ with the same vertex. Recall that $\theta$-labels of vertices do not change. Such assignments produce a diagram $\Delta_{2}$ over $A * B$ without additional irregular vertices because all vertices of $\partial G_{i}$ and $\bar{p}\left(s_{i}\right)$ are regular.

We now identify the path $p\left(u_{i}^{-1}\right)$ with $p\left(u_{i}\right)^{-1}$ and the path $p\left(t_{i}^{-1}\right)$ with $p\left(t_{i}\right)^{-1}$ for every $i=1, \ldots, k$. Doing these identifications, results in a diagram $\Delta_{3}$ over $A * B$ on an oriented surface of genus $k$ such that $\Delta_{3}$ consists of $m+1$ faces $H, G_{1}, \ldots, G_{m}$, $\Delta_{3}$ has a single boundary component, denoted $\partial \Delta_{3}$, and $\Delta_{3}$ contains $\ell$ irregular vertices. The images of paths $p\left(u_{i}\right), p\left(t_{i}\right)$ in $\Delta_{3}$ are denoted $\bar{p}\left(u_{i}\right), \bar{p}\left(t_{i}\right)$, resp., $i=1, \ldots, k$. 
Note that the vertices of paths $\bar{p}\left(u_{i}\right), \bar{p}\left(t_{i}\right)$, different from their end vertices, are all regular and the end vertices of $\bar{p}\left(u_{i}\right), \bar{p}\left(t_{i}\right)$ belong to the boundary path $\partial \Delta_{3}$. We also observe that if $e e^{\prime} \in C\left(\Delta_{3}\right)$ is a corner whose vertex belongs to $\partial \Delta_{3}$ then $\varphi\left(e e^{\prime}\right)=1$. Therefore, we may attach a new face $G_{0}$ such that $\left|\partial G_{0}\right|=\left|\partial \Delta_{3}\right|$ and $\varphi\left(\partial G_{0}\right) \equiv 1^{\left|\partial \Delta_{3}\right|}$ to $\partial \Delta_{3}$ by identifying the paths $\partial G$ and $\partial \Delta_{3}$.

This attachment of $G_{0}$ to $\Delta_{3}$ produces a new diagram $\Delta_{4}$ over $A * B$ such that $\Delta_{4}$ is closed, $\chi\left(\Delta_{4}\right)=2-2 k, \Delta_{4}$ has $\ell$ irregular vertices,

$$
\operatorname{eg}\left(\Delta_{4}\right)=2 k+\ell=\operatorname{mg}\left(w_{0}\right),
$$

$\Delta_{4}$ contains $m$ faces $G_{1}, \ldots, G_{m}$ such that $\varphi\left(\partial G_{i}\right) \equiv_{1} u_{i}$, where $i=1, \ldots, m$, and $\Delta_{4}$ contains two more faces $H, G_{0}$ such that $\varphi(\partial H) \stackrel{*}{=} \varphi\left(\partial G_{0}\right) \stackrel{*}{=} 1$.

Thus we have constructed a closed diagram $\Delta_{4}$ over $A * B$ with some desired properties except for the properties of being reduced and having precisely $m$ faces whose labels are the words $u_{1}, \ldots, u_{m}$.

For a closed diagram $\Delta$ over $A * B$ consider the parameter

$$
\tau(\Delta):=\left(-\chi(\Delta), r_{0}(\Delta),|\Delta(1)|\right),
$$

where as above $\chi(\Delta)$ is the Euler characteristic of $\Delta, r_{0}(\Delta)$ is the number of irregular vertices of $\Delta$, and $|\Delta(1)|$ is the number of nonoriented edges of $\Delta$. We partially order diagrams $\Delta$ over $A * B$ according to their parameters $\tau(\Delta)$ which are ordered lexicographically, i.e., $\tau(\Delta)<\tau\left(\Delta^{\prime}\right)$ if and only if $-\chi(\Delta)<-\chi\left(\Delta^{\prime}\right)$ or $-\chi(\Delta)=-\chi\left(\Delta^{\prime}\right)$ and $r_{0}(\Delta)<r_{0}\left(\Delta^{\prime}\right)$ or $-\chi(\Delta)=-\chi\left(\Delta^{\prime}\right)$ and $r_{0}(\Delta)=r_{0}\left(\Delta^{\prime}\right)$ and $|\Delta(1)|<\left|\Delta^{\prime}(1)\right|$.

Initializing, we set $\widetilde{\Delta}:=\Delta_{4}$ and note that

$$
\tau(\widetilde{\Delta})=(2-2 k, \ell, \widetilde{\Delta}(1)), \quad \operatorname{eg}(\widetilde{\Delta}) \leq \operatorname{mg}\left(w_{0}\right) .
$$

In our inductive arguments below we do not assume that $\widetilde{\Delta}$ is necessarily connected but we do assume that $\widetilde{\Delta}$ has the following property.

(P) Every connected component of a diagram $\Delta$ over $A * B$ contains a face $F$ such that $\varphi(\partial F) \equiv_{1} u_{i}$ for some $i=1, \ldots, m$.

Note that the number of connected components of a diagram $\Delta$ over $A * B$ with property $(\mathrm{P})$ is at most $m$. Hence, $-\chi(\Delta) \geq-2 m$ because $-\chi(\Delta) \geq-2$ whenever $\Delta$ is connected. Since the second and the third components of $\tau(\Delta)$ are nonnegative integers, it follows that there is no strictly decreasing infinite chain

$$
\tau\left(\Delta_{1}\right)>\tau\left(\Delta_{2}\right)>\ldots
$$

in which diagrams $\Delta_{1}, \Delta_{2}, \ldots$ have property $(\mathrm{P})$. This means that we may use induction on parameter $\tau(\Delta)$ in our arguments below if intermediate diagrams, similarly to $\widetilde{\Delta}$, also have property $(\mathrm{P})$.

Now we will make more surgeries over $\widetilde{\Delta}$ aimed to get a reduced diagram.

If $\widetilde{\Delta}$ is reduced and has property $(\mathrm{P})$ then $\widetilde{\Delta}$ is a required diagram and our proof is complete.

Suppose that there is a corner $e f$ of a face $F$ of $\widetilde{\Delta}$ such that $\varphi(e f)=1$. Consider three possible cases.

Case 1: Assume that $e=f^{-1}$, i.e., the vertex $e_{+}=f_{-}$has degree 1 and the corner $e f=e e^{-1}$ is the only corner in $\widetilde{\Delta}$ whose vertex is $e_{+}$. 
If the degree of the vertex $e_{-}$is also 1 then the connected component of $\widetilde{\Delta}$ that contains $e, f$ is a sphere that contains the single face $F$ such that $|\partial F|=2$ and $\varphi(\partial F) \equiv 1 c$, where $c$ is the $\varphi$-label of the second corner of $F$. Since $u_{1}, \ldots, u_{m}$ are cyclically reduced words, it follows that the label of $F$ may not be one of $u_{1}, \ldots, u_{m}$. This contradiction to property $(\mathrm{P})$ of $\widetilde{\Delta}$ proves that the degree of $e_{-}$is greater than 1. Hence, we may take the edges $e, f$ out of $\widetilde{\Delta}$ creating thereby a diagram $\widetilde{\Delta}_{1}$ with property $(\mathrm{P})$ and $\operatorname{eg}\left(\widetilde{\Delta}_{1}\right)=\operatorname{eg}(\widetilde{\Delta})$. The two consecutive corners $e^{\prime} e, e^{-1} f^{\prime}$ of $F$ will disappear and, in their place, we obtain a single corner $e^{\prime} f^{\prime}$ whose $\varphi$-label is defined by $\varphi\left(e^{\prime} f^{\prime}\right):=\varphi\left(e^{\prime} e\right) \varphi\left(f f^{\prime}\right)$, see Fig. 3, where $\varphi\left(e^{\prime} e\right)=a_{1}, \varphi\left(f f^{\prime}\right)=a_{2}$ and $a_{1}, \ldots, a_{4} \in A \cup B$. In view of inequality $\tau\left(\widetilde{\Delta}_{1}\right)<\tau(\widetilde{\Delta})$ and $\operatorname{eg}\left(\widetilde{\Delta}_{1}\right)=\operatorname{eg}(\widetilde{\Delta})$, we can use the induction hypothesis and Case 1 is complete.

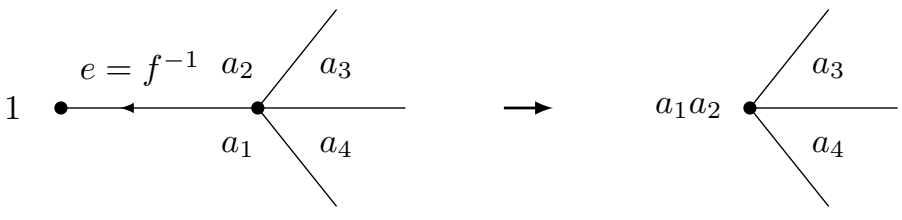

Fig. 3

Case 2: Suppose $e_{-} \neq f_{+}$.

In this case we fold the edges $e$ and $f^{-1}$ within $F$, i.e., we identify $e$ and $f^{-1}$ through the "corner" of $F$ between them. The vertices $e_{-}, f_{+}$become identical and the two corners $e^{\prime} e, f f^{\prime}$ of $F$, whose vertices were $e_{-}, f_{+}$before the fold, turn into a single corner $e^{\prime} f^{\prime}$ whose $\varphi$-label is defined by $\varphi\left(e^{\prime} f^{\prime}\right):=\varphi\left(e^{\prime} e\right) \varphi\left(f f^{\prime}\right)$, see Fig. 4, where $\varphi\left(e^{\prime} e\right)=a_{1}, \varphi\left(f f^{\prime}\right)=a_{5}$ and $a_{1}, \ldots, a_{6} \in A \cup B$. As a result, we obtain a diagram $\widetilde{\Delta}_{1}$ over $A * B$ such that $\tau\left(\widetilde{\Delta}_{1}\right)<\tau(\widetilde{\Delta})$ and $\operatorname{eg}\left(\widetilde{\Delta}_{1}\right)=\operatorname{eg}(\widetilde{\Delta})$. By the induction hypothesis, Case 2 is complete.

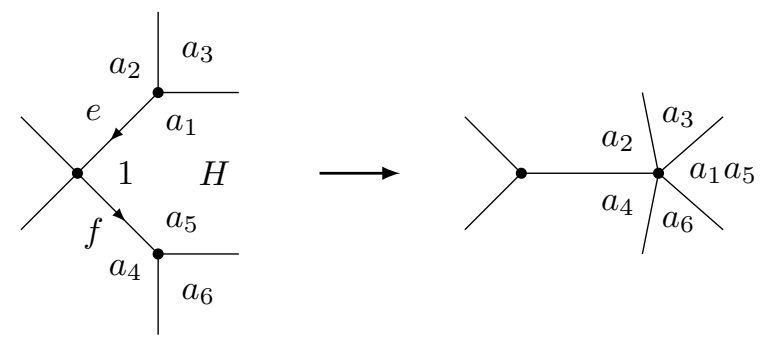

Fig. 4

Case 3: Suppose $e \neq f^{-1}$ and $e_{-}=f_{+}$.

In this case, the path ef is closed and defines a simple closed curve on the underlying surface $S(\widetilde{\Delta})$. We cut $\widetilde{\Delta}$ along this curve and obtain a new diagram $\widetilde{\Delta}_{0}$ with two boundary components, oriented clockwise, which we denote by $e^{\prime} f^{\prime}$ and $\left(e^{\prime \prime} f^{\prime \prime}\right)^{-1}$, where $e^{\prime}, e^{\prime \prime}$ are the images of $e$ in $\widetilde{\Delta}_{0}, f^{\prime}, f^{\prime \prime}$ are the images of $f$ in $\widetilde{\Delta}_{0}$, and $e^{\prime} f^{\prime}$ is the image of the corner $e f$ of $H$ in $\widetilde{\Delta}_{0}$, see Fig. 5 . 


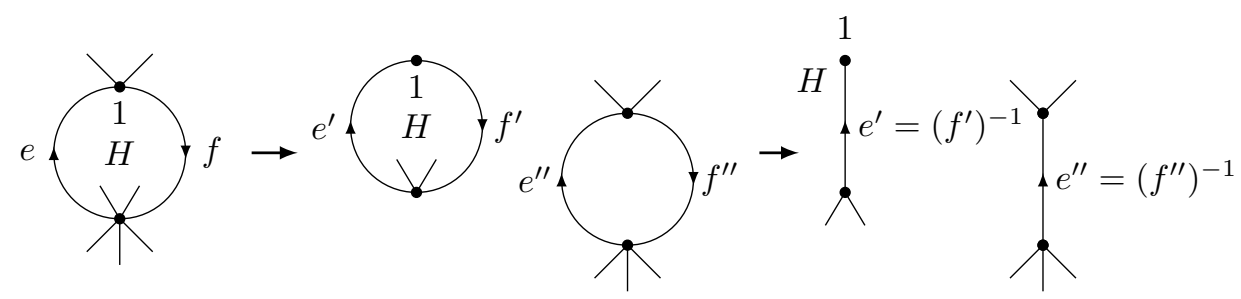

Fig. 5

Note that $\chi\left(\widetilde{\Delta}_{0}\right)=\chi(\widetilde{\Delta})$ and the closed paths $e^{\prime} f^{\prime}, e^{\prime \prime} f^{\prime \prime}$ might belong to different connected components of $\widetilde{\Delta}_{0}$ which happens when ef defines a separating curve on $S(\widetilde{\Delta})$.

We identify the edges $e^{\prime}$ and $\left(f^{\prime}\right)^{-1}$ and the edges $e^{\prime \prime}$ and $\left(f^{\prime \prime}\right)^{-1}$ thus eliminating the boundary of $\widetilde{\Delta}_{0}$. The result is a closed diagram $\widetilde{\Delta}_{1}$ over $A * B$ such that

$$
\chi\left(\widetilde{\Delta}_{1}\right)=\chi\left(\widetilde{\Delta}_{0}\right)+2=\chi(\widetilde{\Delta})+2 .
$$

Observe that the images of vertices $e_{-}^{\prime}, e_{-}^{\prime \prime}$ in $\widetilde{\Delta}_{1}$ could be both irregular even if $e_{-}$is regular in $\widetilde{\Delta}$, the image of $e_{+}^{\prime}$ in $\widetilde{\Delta}_{1}$ is regular and the image of $e_{+}^{\prime \prime}$ in $\widetilde{\Delta}_{1}$ is regular if and only if $e_{+}$is regular in $\widetilde{\Delta}$. This means that $r_{0}\left(\widetilde{\Delta}_{1}\right) \leq r_{0}(\widetilde{\Delta})+2$. Therefore, we have

$$
\operatorname{eg}\left(\widetilde{\Delta}_{1}\right)=2-\chi\left(\widetilde{\Delta}_{1}\right)+r_{0}\left(\widetilde{\Delta}_{1}\right) \leq \operatorname{eg}(\widetilde{\Delta}) .
$$

Note that $\widetilde{\Delta}_{1}$ might have a connected component $\widetilde{\Delta}_{1,1}$ with the property that $\varphi(\partial G) \stackrel{*}{=} 1$ for every face $G$ in $\widetilde{\Delta}_{1,1}$, i.e., $\widetilde{\Delta}_{1}$ might lack the property $(\mathrm{P})$. Since $\widetilde{\Delta}$ has property $(\mathrm{P})$, it follows that there is at most one such component $\widetilde{\Delta}_{1,1}$ in $\widetilde{\Delta}_{1}$. If $\widetilde{\Delta}_{1,1}$ does exist then we take $\widetilde{\Delta}_{1,1}$ out of $\widetilde{\Delta}_{1}$ and denote thus obtained diagram $\widetilde{\Delta}_{2}$. If $\widetilde{\Delta}_{1,1}$ does not exist then we set $\widetilde{\Delta}_{2}:=\widetilde{\Delta}_{1}$. Clearly, $\widetilde{\Delta}_{2}$ has property $(\mathrm{P})$.

First we consider the subcase when either $\widetilde{\Delta}_{1,1}$ does not exist or $\widetilde{\Delta}_{1,1}$ exists and $\chi\left(\widetilde{\Delta}_{1,1}\right) \leq 0$. Since $\chi\left(\widetilde{\Delta}_{1}\right)=\chi\left(\widetilde{\Delta}_{2}\right)+\chi\left(\widetilde{\Delta}_{1,1}\right)$, it follows from the definitions and the inequality (5) that $-\chi\left(\widetilde{\Delta}_{2}\right) \leq-\chi(\widetilde{\Delta})-2$ and $\operatorname{eg}\left(\widetilde{\Delta}_{2}\right) \leq \operatorname{eg}(\widetilde{\Delta})$. Hence, $\tau\left(\widetilde{\Delta}_{2}\right)<\tau(\widetilde{\Delta})$ and, by the induction hypothesis, this subcase is complete.

Now assume that $\widetilde{\Delta}_{1,1}$ exists and $\chi\left(\widetilde{\Delta}_{1,1}\right)>0$. Since $\widetilde{\Delta}_{1,1}$ is oriented and connected, it follows that $\chi\left(\widetilde{\Delta}_{1,1}\right)=2$ and $\widetilde{\Delta}_{1,1}$ is a sphere. Since $\chi\left(\widetilde{\Delta}_{1}\right)=\chi\left(\widetilde{\Delta}_{2}\right)+$ $\chi\left(\widetilde{\Delta}_{1,1}\right)$, we have $\chi\left(\widetilde{\Delta}_{2}\right)=\chi(\widetilde{\Delta})$.

Let us show that $r_{0}\left(\widetilde{\Delta}_{2}\right) \leq r_{0}(\widetilde{\Delta})$. It follows from our construction that either $r_{0}\left(\widetilde{\Delta}_{1}\right)=r_{0}(\widetilde{\Delta})$ or $r_{0}\left(\widetilde{\Delta}_{1}\right)=r_{0}(\widetilde{\Delta})+2$. If $r_{0}\left(\widetilde{\Delta}_{1}\right)=r_{0}(\widetilde{\Delta})$ then $r_{0}\left(\widetilde{\Delta}_{2}\right) \leq r_{0}(\widetilde{\Delta})$ as desired. Assume that $r_{0}\left(\widetilde{\Delta}_{1}\right)=r_{0}(\widetilde{\Delta})+2$. Then it follows from the definitions that $r_{0}\left(\widetilde{\Delta}_{1,1}\right) \geq 1$ because the image of the vertex $e_{-}^{\prime}$ in $\widetilde{\Delta}_{1,1}$ is irregular. It is not difficult to show (e.g., by induction on $\left.\left(\left|\widetilde{\Delta}_{1,1}(2)\right|,\left|\widetilde{\Delta}_{1,1}(1)\right|\right)\right)$ that the equality $r_{0}\left(\widetilde{\Delta}_{1,1}\right)=1$ is impossible. Therefore, $r_{0}\left(\widetilde{\Delta}_{1,1}\right) \geq 2$ and we can conclude that

$$
r_{0}\left(\widetilde{\Delta}_{2}\right) \leq r_{0}\left(\widetilde{\Delta}_{1}\right)-2 \leq r_{0}(\widetilde{\Delta})
$$

as desired. 
Since $\left|\widetilde{\Delta}_{2}(1)\right|+\left|\widetilde{\Delta}_{1,1}(1)\right|=|\widetilde{\Delta}(1)|$ and $\left|\widetilde{\Delta}_{1,1}(1)\right|>0$, it follows from $\chi\left(\widetilde{\Delta}_{2}\right)=$ $\chi(\widetilde{\Delta})$ and $r_{0}\left(\widetilde{\Delta}_{2}\right) \leq r_{0}(\widetilde{\Delta})$ that $\tau\left(\widetilde{\Delta}_{2}\right)<\tau(\widetilde{\Delta})$. It is also clear that $\operatorname{eg}\left(\widetilde{\Delta}_{2}\right) \leq \operatorname{eg}(\widetilde{\Delta})$, hence, by the induction hypothesis, Case 3 is complete.

Thus in all Cases $1-3$ we have been able to construct a diagram $\widetilde{\Delta}^{\prime}$ over $A * B$ such that $\operatorname{eg}\left(\widetilde{\Delta}^{\prime}\right) \leq \operatorname{eg}(\widetilde{\Delta}), \tau\left(\widetilde{\Delta}^{\prime}\right)<\tau(\widetilde{\Delta})$ and $\widetilde{\Delta}^{\prime}$ contains $m$ faces $G_{1}, \ldots, G_{m}$ such that $\varphi\left(\partial G_{i}\right) \equiv_{1} u_{i}, i=1, \ldots, m$. This completes the proof of Lemma 1 .

\section{CAR Motions}

This Section is similar to a corresponding section of [12] and contains necessary definitions and statements of [20, 21] with some simplifications.

Consider a closed map $\Delta$ on a closed oriented compact surface. A car moving around a face $F$ of $\Delta$ is an orientation preserving covering of the boundary path $\partial F$ of $F$ by an oriented circle $C=\mathbb{R} / M \mathbb{Z}$ called the circle of time and regarded as the quotient of the real numbers $\mathbb{R}$ by its subgroup $M \mathbb{Z}$, where $\mathbb{Z}$ is the set of integers and $M \in \mathbb{R}$.

Informally, a car is a point moving along the boundary path of a face in counterclockwise direction (the interior of the face remains on the left) without U-turns and stops. The motion is periodic.

The degree of a vertex $v$ of a map $\Delta$ is the number of oriented edges of $\Delta$ whose terminal vertex is $v$. By the definition, a point in the interior of an edge of $\Delta$ has degree two.

Let $v$ be a point of the 1-skeleton $\Delta[1]$ of $\Delta$ and suppose that the number of cars being at a moment of time $t$ at the point $v$ is equal to the degree of $v$. Then $v$ is called a point of complete collision.

A multiple car motion of period $T$ on $\Delta$ is a set of cars $\alpha_{F, j}: C \rightarrow \partial F$, defined for every face $F$ of $\Delta$ and for every $j=1, \ldots, d_{F}$, where $d_{F} \geq 1$ is an integer, such that the following hold true.

(M1) If $d_{F}>1$ then $\alpha_{F, j}(t+T)=\alpha_{F, j+1}(t)$ for every $t \in \mathbb{R}$ and $j \in\left\{1, \ldots, d_{F}\right\}$, here the second subscripts are modulo $d_{F}$ and addition of points of $C$ is defined according to $C=\mathbb{R} / M \mathbb{Z}$, where $M$ is an integer multiple of $T$.

(M2) For every face $F$ of $\Delta$, there exists a partition of $\partial F$ into $d_{F}$ consecutive arcs with disjoint interiors such that, during the time interval $[0, T]$, each car $\alpha_{F, j}$ is moving along the $j$ th arc of the partition.

Lemma 2 (20, 21]). For every multiple car motion defined on a closed map $\Delta$ on an oriented compact surface, the number of points of complete collision is at least

$$
\chi(\Delta)+\sum_{F \in \Delta(2)}\left(d_{F}-1\right),
$$

where the summation runs over all faces $F$ of $\Delta$.

We remark that, in articles 20, 21, Lemma 2 is stated and proved for connected surfaces, but it remains valid in nonconnected case because both parts of the inequality in Lemma 2 are additive under disjoint union.

\section{Proof of Theorem}

First we note that an arbitrary free product $\mathcal{F}=\underset{\alpha \in I}{*} G_{\alpha}$ of nontrivial groups $G_{\alpha}$, where $|I|>1$, can be embedded into a free product $A * B$ of two groups $A, B$ 
by means of a monomorphism $\mu: \mathcal{F} \rightarrow A * B$ in such a way that the following properties (E1) $-(\mathrm{E} 2)$ hold true.

(E1) If $w \in \mathcal{F}$ is a reduced word then $\mu(w) \in A * B$ is also reduced and the set of finite orders of letters of $w$ is identical to that of $\mu(w)$.

(E2) An element $w \in \mathcal{F}$ is conjugate in $\mathcal{F}$ to an element of a free factor $G_{\alpha}$ if and only if $\mu(w)$ is conjugate in $A * B$ to an element of $A \cup B$.

Indeed, let $A:=\mathcal{F}$ and let $B:=F\left(b_{\alpha} ; \alpha \in I\right)$ be a free group whose free generators are letters $b_{\alpha}, \alpha \in I$. Then the desired embedding $\mu: \mathcal{F} \rightarrow A * B$ can be defined by extending to $\mathcal{F}$ the map $\mu(g):=b_{\alpha}^{-1} g b_{\alpha}$ for every $g \in G_{\alpha}$. It is easy to see that both properties (E1) and (E2) hold true.

Observe that if $w \in \mathcal{F}$ then it follows from property (E2) that

$$
\mathrm{qp}(w) \leq \mathrm{qp}(\mu(w)) \quad \text { and } \quad \operatorname{mg}(\mu(w)) \leq \mathrm{mg}(w) .
$$

Hence, in view of property (E1), it suffices to prove our Theorem for the free product $A * B$ of two factors $A, B$.

Let $w \in A * B$ be a word such that $\mathrm{qp}(w)$ is finite and consider a quasiperiodic factorization for $w$ of the form

$$
w \stackrel{*}{=} s_{1} u^{n_{1}} s_{1}^{-1} s_{2} u^{n_{2}} s_{2}^{-1} \ldots s_{m} u^{n_{m}} s_{m}^{-1},
$$

where $u$ is a cyclically reduced word, $s_{j} \in A * B, n_{j}>0$, and $\operatorname{qp}(w)=\sum_{j}\left(n_{j}-1\right)$. By Lemma 1 , there exists a reduced diagram $\Delta$ over $A * B$ such that $\Delta$ contains precisely $m$ faces $F_{1}, \ldots, F_{m}$ whose labels are the words $u^{n_{1}}, u^{n_{2}}, \ldots, u^{n_{m}}$, resp., and

Denote

$$
\operatorname{eg}(\Delta) \leq \operatorname{mg}(w)
$$

$u \equiv a_{1} b_{1} \ldots a_{r} b_{r}$,

where $a_{i} \in A, b_{i} \in B$ and $a \neq 1, b \neq 1$.

We will now define a multiple car motion on $\Delta$ in the following manner. For every $j=1, \ldots, m$, there are $n_{j}$ cars that move around the boundary path $\partial F_{j}$, where $\varphi\left(\partial F_{j}\right) \equiv u^{n_{j}}$, with constant speed, one edge per unit of time, and, at the initial moment of time, $t=0$, the cars are located at distinct corners whose $\varphi$-labels are $b_{r}$, here $b_{r}$ means the last letter of $u$, see (7). It is easy to see that this is a periodic motion with period $2 r$. By Lemma 2 , there are at least $\chi(\Delta)+\sum_{j}\left(n_{j}-1\right)$ points of complete collision in $\Delta$.

Let us analyze where these complete collisions may occur.

First, note that a complete collision may not occur at an interior point of an edge of $\Delta$. Indeed, at every even moment of time $t=2 i$, where $i \in \mathbb{Z}$, all cars are located at $B$-vertices, while at every odd moment of time $t=2 i+1$ all cars are located at $A$-vertices. Therefore, during the time interval $(2 i, 2 i+1)$ every car is moving from a $B$-vertex to an $A$-vertex, while during the time interval $(2 i-1,2 i)$ every car is moving from an $A$-vertex to a $B$-vertex. Thus any two cars are never moving along the same edge in opposite directions and may not collide in the interior of an edge.

Second, observe that a complete collision may not occur at a regular vertex. To prove this claim, we note that at every integer moment of time all cars are located at corners with the same $\varphi$-label, as denoted in (7). More specifically, at an even moment of time $t=2 i$, where $i \in \mathbb{Z}$, all cars are located at corners with $\varphi$-label being $b_{i}$, as indicated in (7), here indices are modulo $r$, and, at an odd moment of time $t=2 i+1$, all cars are located at corners with $\varphi$-label being $a_{i}$, as denoted in 
(17). Therefore, all corners, whose vertex $v$ is a given point of a complete collision, must have the same $\varphi$-label, as indicated in the factorization (7). If $v$ is a regular vertex of degree $d$ then it follows from the definition of a regular vertex that $a_{i}^{d} \stackrel{A}{=} 1$ or $b_{i}^{d} \stackrel{B}{=} 1$ for some $i$. Since $d$ does not exceed the number of all corners in $\Delta$ with $\varphi$-label $a_{i}$ or $b_{i}$, it follows that $d \leq \sum_{j} n_{j}$. However, this inequality contradicts the assumption that every letter of $u$ has order greater than $\sum_{j} n_{j}$. This contradiction proves our claim.

Therefore, complete collisions can only occur at irregular vertices of $\Delta$. Recall that, by Lemma 2 , there are at least $\chi(\Delta)+\sum_{j}\left(n_{j}-1\right)$ points of complete collision in $\Delta$. Hence, we conclude that the number of irregular vertices of $\Delta$ is at least $\chi(\Delta)+\sum_{j}\left(n_{j}-1\right)$, i.e.,

$$
r_{0}(\Delta) \geq \chi(\Delta)+\sum_{j}\left(n_{j}-1\right) .
$$

Therefore, it follows from the inequality (6) that

$$
\mathrm{qp}(w)=\sum_{j}\left(n_{j}-1\right) \leq-\chi(\Delta)+r_{0}(\Delta)=\operatorname{eg}(\Delta)-2 \leq \operatorname{mg}(w)-2,
$$

as required. This completes the proof of Theorem.

Corollaries are straightforward from the definitions and Theorem.

Acknowledgments. The authors thank Lvzhou Chen and Danny Calegari for helpful discussion and remarks. The authors are grateful to the referee for useful suggestions.

\section{REFERENCES}

[1] G. Baumslag and A. Steinberg, Residual nilpotence and relations in free groups, Bull. Amer. Math. Soc. 70(1964), 283-284.

[2] L. Chen, Spectral gap of scl in free products, Proc. Amer. Math. Soc. 146(2018), 3143-3151. See also arXiv:1611.07936 [math.GT].

[3] A. Clifford and R. Z. Goldstein, Tesselations of $S^{2}$ and equations over torsion-free groups, Proc. Edinburgh Math. Soc. 38(1995), 485-493.

[4] A. Clifford and R. Z. Goldstein, Equations with torsion-free coefficients, Proc. Edinburgh Math. Soc. 43(2000), 295-307.

[5] M. M. Cohen and C. Rourke, The surjectivity problem for one-generator, one-relator extensions of torsion-free groups, Geometry \& Topology 5(2001), 127-142. See also arXiv:math.GR/0009101.

[6] J. A. Comerford, L. P. Comerford and C. C. Edmunds, Powers as products of commutators, Comm. Algebra 19(1991), 675-684.

[7] L. P. Comerford, C. C. Edmunds, and G. Rosenberger, Commutators as powers in free products of groups, Proc. Amer. Math. Soc. 122(1994), 47-52.

[8] M. Culler, Using surfaces to solve equations in free groups, Topology 20(1981), 133-145.

[9] A. J. Duncan and J. Howie, The genus problem for one-relator products of locally indicable groups, Math. Z. 208(1991), 225-237.

[10] R. Fenn and C. Rourke, Klyachko's methods and the solution of equations over torsion-free groups, L'Enseignment Math. 42(1996), 49-74.

[11] R. Fenn and C. Rourke Characterisation of a class of equations with solution over torsionfree groups, "The Epstein Birthday Schrift", (ed. by I. Rivin, C. Rourke and C. Series), Geometry and Topology Monographs 1(1998), 159-166.

[12] E. V. Frenkel and Ant. A. Klyachko, Commutators cannot be proper powers in metric small-cancellation torsion-free groups, preprint, arXiv:1210.7908 [math.GR].

[13] M. Forester and C. Rourke, Diagrams and the second homotopy group, Comm. Anal. Geom. 13(2005), 801-820. See also arXiv:math.AT/0306088 
[14] R. I. Grigorchuk and P. F. Kurchanov, On the width of elements in free groups, Ukrainian Math. J. 43(1991), 911-918.

[15] J. Howie, The solution of length three equations over groups, Proc. Edinburgh Math. Soc. 26(1983), 89-96.

[16] J. Howie, The quotient of a free product of groups by a single high-powered relator. II. Fourth powers, Proc. London Math. Soc. 61(1990), 33-62.

[17] S. V. Ivanov, The bounded and precise word problems for presentations of groups, preprint, arXiv:1606.08036 [math.GR].

[18] S. V. Ivanov and Ant. A. Klyachko, The asphericity and Freiheitssatz for certain LOTpresentations of groups, Internat. J. Algebra Comp. 11(2001), 291-300.

[19] Ant. A. Klyachko, A funny property of a sphere and equations over groups, Comm. Algebra 21(1993), 2555-2575.

[20] Ant. A. Klyachko, Asphericity tests, Internat. J. Algebra Comp. 7(1997), 415-431.

[21] Ant. A. Klyachko The Kervaire-Laudenbach conjecture and presentations of simple groups, Algebra and Logic 44(2005), 219-242. See also arXiv:math.GR/0409146.

[22] Ant. A. Klyachko, How to generalize known results on equations over groups, Mathematical Notes 79(2006), 377-386. See also arXiv:math.GR/0406382

[23] Ant. A. Klyachko, The SQ-universality of one-relator relative presentations, Sbornik Mathematics 197(2006), 1489-1508. See also arXiv:math.GR/0603468

[24] Ant. A. Klyachko, Free subgroups of one-relator relative presentations, Algebra and Logic 46(2007), 158-162. See also arXiv:math.GR/0510582

[25] Ant. A. Klyachko, The structure of one-relator relative presentations and their centres, J. Group Theory 12(2009), 923-947. See also arXiv:math.GR/0701308

[26] Ant. A. Klyachko and D. E. Lurye, Relative hyperbolicity and similar properties of onegenerator one-relator relative presentations with powered unimodular relator, J. Pure Appl. Algebra 216(2012), 524-534. See also arXiv:1010.4220

[27] Le Thi Giang, The relative hyperbolicity of one-relator relative presentations, J. Group Theory 12(2009), 949-959. See also arXiv:0807.2487

[28] R. C. Lyndon and P. E. Schupp, Combinatorialgroup theory, Springer-Verlag, 1977.

[29] A. Yu. Ol'shanskii, Geometry of defining relations in groups, Nauka, Moscow, 1989; English translation: Math. and Its Applications, Soviet series, vol. 70, Kluwer Acad. Publ., 1991.

[30] A. Yu. Ol'shanskii, On calculation of width in free groups, London Math. Soc. Lecture Note Ser. 204(1995), 255-258.

[31] M. P. Schützenberger, Sur l'equation $a^{2+n}=b^{2+m} c^{2+p}$ dans un groupe libre, C. R. Acad. Sci. Paris 248(1959), 2435-2436.

Department of Mathematics, University of Illinois, Urbana, IL 61801, U.S.A.

E-mail address: ivanov@illinois.edu

Department of Mechanics and Mathematics, Moscow State University, Moscow, 119991, Russia

E-mail address: klyachko@mech.math.msu.su 